\title{
Publisher's Note: Direct Imaging of Dynamic Glassy Behavior in a Strained Manganite Film \\ [Phys. Rev. Lett. 115, 265701 (2015)]
}

\author{
Worasom Kundhikanjana, Zhigao Sheng, Yongliang Yang, Keji Lai, Eric Yue Ma, Yong-Tao Cui, \\ Michael A. Kelly, Masao Nakamura, Masashi Kawasaki, Yoshinori Tokura, Qiaochu Tang, \\ Kun Zhang, Xinxin Li, and Zhi-Xun Shen \\ (Received 30 December 2015; published 7 January 2016)
}

DOI: 10.1103/PhysRevLett.116.019904

This paper was published online on 23 December 2015 with an omission of an author's affiliation and of an acknowledgment on page 5. The author list and affiliation list should read as

Worasom Kundhikanjana ${ }^{1,2}$, Zhigao Sheng ${ }^{3,4,5}$, Yongliang Yang $^{1}$, Keji Lai ${ }^{6}$, Eric Yue Ma ${ }^{1}$, Yong-Tao Cui ${ }^{1}$, Michael A. Kelly ${ }^{1}$, Masao Nakamura ${ }^{3}$, Masashi Kawasaki, ${ }^{3,7}$, Yoshinori Tokura ${ }^{3,7}$, Qiaochu Tang ${ }^{8}$, Kun Zhang, Xinxin $\mathrm{Li}^{8}$, and Zhi-Xun Shen ${ }^{1, *}$

${ }^{1}$ Department of Applied Physics and Geballe Laboratory for Advanced Materials, Stanford University, Stanford, California 94305, USA

${ }^{2}$ School of Physics, Institute of Science, Suranaree University of Technology, Nakorn Ratchasima, Thailand

${ }^{3}$ RIKEN Center for Emergent Matter Science (CEMS), Wako 251-0198, Japan

${ }^{4}$ High Magnetic Field Laboratory of Chinese Academy of Science, Hefei 230031, China

${ }^{5}$ Collaborative Innovation Center of Advanced Microstructures, Nanjing University, Nanjing 210093, China

${ }^{6}$ Department of Physics, University of Texas at Austin, Austin, Texas 78712, USA

${ }^{7}$ Department of Applied Physics and Quantum Phase Electronics Research Center (QPEC), University of Tokyo, Tokyo 113-8656, Japan

${ }^{8}$ State Key Lab of Transducer Technology, Shanghai Institute of Microsystem and Information Technology, Chinese Academy of Sciences, Shanghai 200050, China

On page 5, the last sentence of the Acknowledgments should read as "This work was supported by the Natural Science Foundation of China, Grant No. 11574316, No. U1532155, and the One Thousand Talents Program of China." The paper has been corrected as of 30 December 2015. The text is correct in the printed version of the journal. 